\title{
Multi-Agent Based SMART Metering AND MONITORING OF POWER DisTRIBUTION SYSTEM: AN EXTENDED FRAMEWORK
}

\author{
M. K. Ahmed ${ }^{1}$, Bala Modi ${ }^{2}$ and $S$. O. Oladejo ${ }^{3}$ \\ 1,2,3 Department of Mathematics, Gombe State University, Gombe, Nigeria
}

\begin{abstract}
One of the problems faced by the Electricity Power consumers is the issue of charging higher than their consumption. The extended framework presented in this work provides a lasting solution by developing a Multi-agent Based System, which allows a Meter Agent to detect a case of bypassing a prepaid meter and report the case to the distribution company. Similarly, the system should be able monitor the amount in which the customer is being charged based on their power consumption. This will solve the problem of overcharging power consumers. Multi-Agent System Engineering (MaSE) methodology was used to establish how agents are able to accomplish the stated challenge encountered in the Nigerian Electricity Distribution System. The proposed platform shows that Multi-Agent Systems can play a vital role in addressing the challenges facing the power distribution sector.
\end{abstract}

\section{KEYWORDS}

Agent, Multi-Agent System, Power Distribution System, Multi-agent Software Engineering (MaSE), Distributed Systems

\section{INTRODUCTION}

Agent-based computing is one of the most powerful technologies in used in developing and deploying distributed complex systems [15]. Agents were presented in [5] as a new paradigm for software development since the inception of object-oriented designs. Today, the concept of intelligent agents is used in diverse range of applications such as: manufacturing, real-time control systems, electronic commerce, network management, transportation systems, information management, scientific computing, health care, and entertainment, etc. The reason for the growing success of agent oriented paradigm in these areas of application is that the inherent distribution method allows for a natural decomposition of the system into multiple agents that interact with each other to achieve a desired global objective.

Agents can operate without the direct intervention of humans or others [13]. This feature helps agents to monitor and detect fault in a power distribution system of a given area. In Multi-Agent System (MAS), the agents communicate with other agents in a system to achieve a collective goal. Agents can also perceive their environment and respond in a timely fashion to environmental changes [9].

The authors in [5] defined multi-agent system as a group of agents which sense the environment and act in order to achieve specific objectives. MAS can therefore be seen as a network of interacting software modules that bring together dispersed systems that collectively manage complex tasks that are beyond the capacity of any individual system on the network. 
The authors in [14] described the term multi-agent system as implying more than one agent interacting with each other within an underlying communication infrastructure where individual agents are often distributed and autonomous. Multi-agent systems are based on the idea that a cooperative working environment can cope with problems which are hard to solve using the traditional centralized approach to computation. Agents are used to interact in a flexible and dynamic way to solve problems more efficiently. The rest of the paper is arranged thus: Section describes the various related works and problem statement as perceived and presented by other authors, while Section 3 describes the framework of the design in this paper. Section 4 discusses the preliminary results obtained and Section 5 is the conclusion drawn from the paper.

\section{RELATED WORKS}

Concerning the Agent-Oriented Platform design of this paper, a number of works were reviewed to know the status of MAS platforms in relation to power distribution systems [12], [6], [4], [10], [3] and [14]. Additionally, Agent-Oriented Software Methodologies [7] and [11] were also reviewed. The purpose of this review is to select the most appropriate and suitable agent platform and agent-oriented software methodology for this work.

The conceptual structure described in [6], is a structure for a power system self-healing infrastructure which was analyzed. Agents [1] have hierarchical levels and higher level agents coordinating in a group of lower level agents. Also, all agents [8] are deemed to have their distinct responsibilities e.g. acquiring data, analyzing data, managing situations etc. In other words, each step of decision making is done by a special agent.

The authors in [12] and [4], offered a MAS design for distribution systems restoration. The paper presented network buses as agents, along with a facilitation agent responsible for aiding negotiation processes among bus agents. An intelligent agent-based environment that coordinates maintenance schedules was introduced in [10] and an autonomous regional active network management system was presented and discussed as well in [3].In broader terms, all the consulted literatures so far consists of a master agent which makes the final decision based on the data received from other agents. However, this cannot be considered distributed system, since the master agent behaves like a control center.

An agent committee approach was presented in [14] to push the technology further in solving power restoration problems in a more distributed and efficient way. The idea is to allow neighboring switch agents to be organized into a local power committee. The responsibility of the committee is to ensure that the local power demand can be satisfied as well as reconciled. The reconciliation is with an optimal solution to the conflicting issues proposed among committee members who are responsible for identifying a feasible power source to the local power demand problems. The advantage of this approach is to localize the global problem solving ability to a local committee of agents who could coordinate with neighboring committees to achieve an agreement. The agreement is one that satisfies the global objective of the distribution system without relying on a pre-determined centralized optimization algorithm as other traditional approaches do. The committee-based multi-agent system's [14] objective is to find a solution of power restoration problem that can maximize the service zones while minimizing the number of switch operations under the topological and operational constraints of power distribution systems. While the approach in [14] contributed by taking this new technology to a higher level, but since a committee is an organization of agents, it still needed a master to essentially conduct the decisions of the committee and communicate with its committee members and other committees. As such, the system will encounter a problem whenever a break in communication occurs 
between the Local Power Committee leader and the central unit of the system. This problem was addressed in [17] in which a Multi-agent System that enable agents to detect and report fault in Power Distribution System (PDS) with the minimum time delay was demonstrated. Multi-agent System Engineering (MaSE) methodology was used to describe how the agents will be managing the complexity of PDS environment. The simulation of the framework was done using MatLab/Simulink. The result indicates that Agents Oriented System technology can be used to manage fault in Power Distribution System with the minimum time delay.

In Our previous work that is been extended here [16], we presented a Multi-agent System platform that allows agents to detect and report fault in Power Distribution System (PDS) with the minimum time delay presented. Multi-agent System Engineering (MaSE) methodology was used to demonstrate how agents will be able to manage the complexity of PDS. The simulation of the framework was done using Java Agent Development Framework (JADE). However, a lot needed to be done to address the problem of overcharging power consumers beyond their power consumption status (Customer Challenge) and find a way to detect meter bypassing by the power consumers to avoid being charged (Distribution Companies Challenge).

\subsection{Problem Statement}

The major problems faced by the Electricity Distribution Companies in Nigeria detection of illegal connection, fault detection. Similarly on the side of the consumer, there are so much complains overcharging. Even though the work in [16] already addressed the problem of fault detection and illegal connections, there is still the need to extend the process in order to tackle the problem of overcharging customers beyond their power consumption rates and to also find a way of detecting cases of bypassing prepaid meters using an Agent (Smart Meters). This is the focus of this paper.

\section{Multi-Agent BaSEd System FrameWOrK 3.1 THe Switch AGENT}

Switch agents are passive agents who sense their environments and forward the information gathered to their Zone Agents along the topology. They are in charge of maintaining the flow of current in their areas in a normal state. Therefore, they must periodically update their knowledge of the area in order to adapt their power injection.

These agents are responsible for monitoring the system voltage and frequency in order to detect contingency situations or grid failures, and end up sending signals to the zone agent when a fault is detected. In addition to that, the switch agent is also responsible for load termination in case of high voltage to avoid damage of consumer appliances.

The goal of each switch agent is to update the topological and electrical information about the actual area whose initiating switch agent is the master. Figure 1 depicts switch agent architecture. In the architecture, the agent checks each active adjacent link and sends message information to its zone agent about the current states of attached links and waits for a response. The Zone Agent as described in Section 3.4 receives this message and sends an acknowledgement to the switch agent and recursively forwards it further to the control center also described in Section 3.3. In case there is no acknowledgement from the zone agent receiving the message, the switch agent will resend the same message to its zone agent for further action. 
International Journal of Artificial Intelligence and Applications (IJAIA), Vol.10, No.1, January 2019

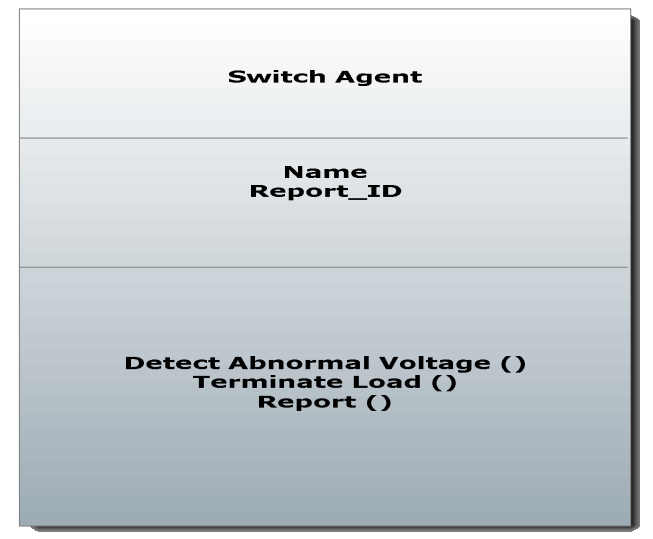

Figure 1. Switch Agent (Micro Level) Architecture.

\subsection{Monitoring Agent}

The Monitoring Agent (Fault Agent) is responsible for monitoring the distribution line in order to identify fault (line breakage) and report same to its zone agent. Figure 2 below shows the agent micro level architecture.

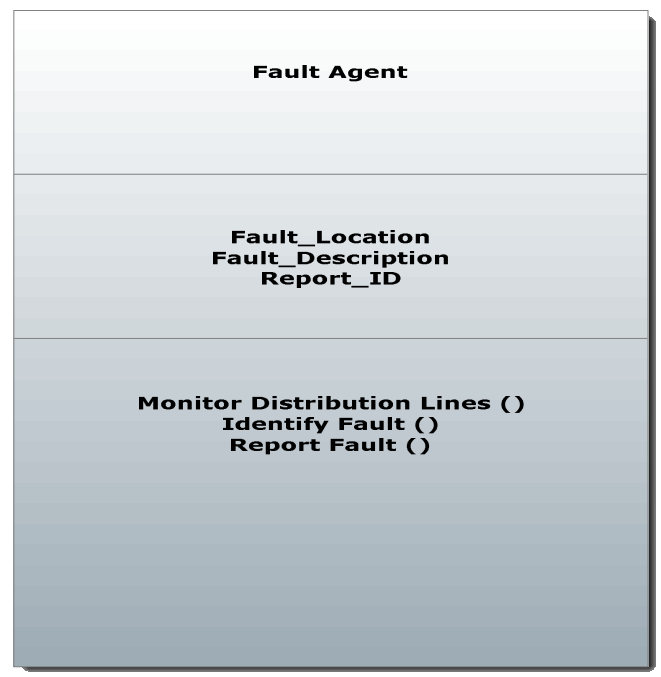

Figure 2. Monitoring Agent (Micro Level) Architecture.

\subsection{CONNECTION AGENT}

One of the major problems faced by Electricity Distribution Companies is that of illegal connections. This Connection Agent is responsible for detecting such connections, reporting same to its zone agent and terminating the illegal connection. Figure 3 below shows the agent micro level architecture. 


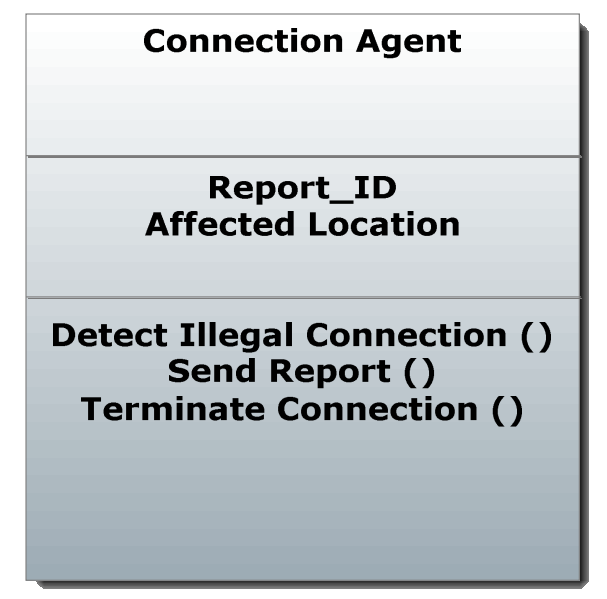

Figure 3. Connection Agent (Micro Level) Architecture.

\subsection{The Zone AgenT}

At the beginning, each Zone Agent knows and contains only the data of the Switch Agents located in the zone. As the process proceeds, Zone Agents gain additional data from the Switch Agents and use this information fulfill the following goals:

I. Determine where the faults occurred in the zone using the message received from Switch Agents.

II. Sending acknowledgements to the switch agents sending the message

III. Forwarding the messages received to the control center and await acknowledgement.

Figure 4 below depicts single zone agent architecture.

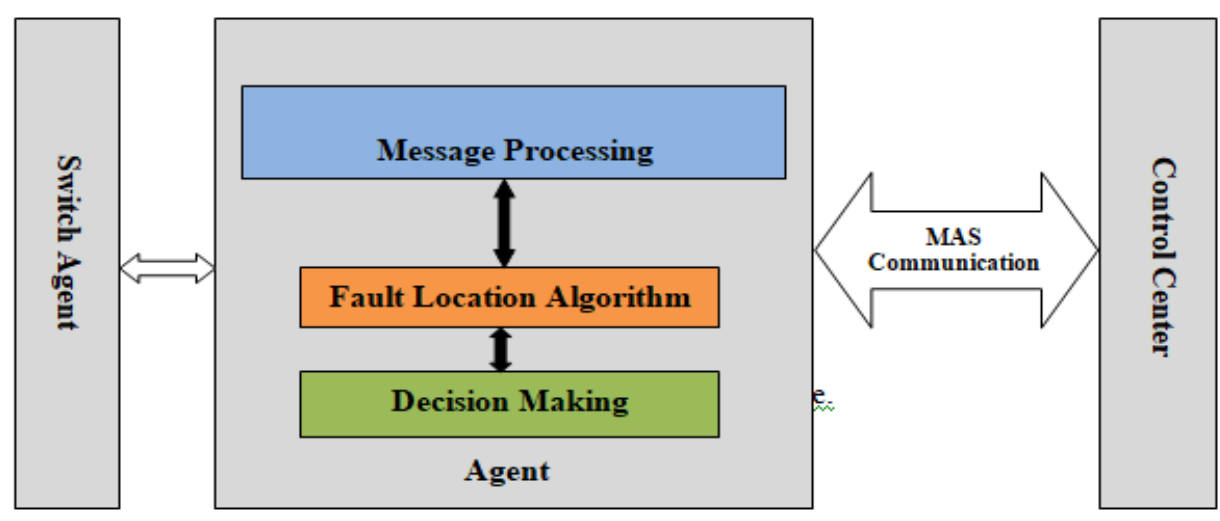

Figure 4. Single Zone Agent Architecture.

\subsection{MeTER AGENT}

One of the major complains by the electricity consumers is that of are overcharging of their power consumption by the power companies. The Meter Agent described in this paper is one of the additional agents from our previous work. The job of this agent is to ensure that power customers are charged according to their consumptions. The agent is responsible for keeping 
records of the energy charged for verification by both the customer and the national electricity regulatory commission. This agent is a mobile agent that will help in resolving disputes between the National Electricity Regulatory Commission (NERC), power consumer (customers) and Distribution Company. The agent's internal architecture is shown in Figure 5. The agent is capable of starting and stopping operation (i.e. charging customer when consuming electrical power), reporting inactivity and reporting alteration in the case of bypassing and other related scenario. The report sent by the individual prepaid meter is received by both NERC and Distribution Company for resolving dispute as depicted by the flowchart shown in Figure 6 .

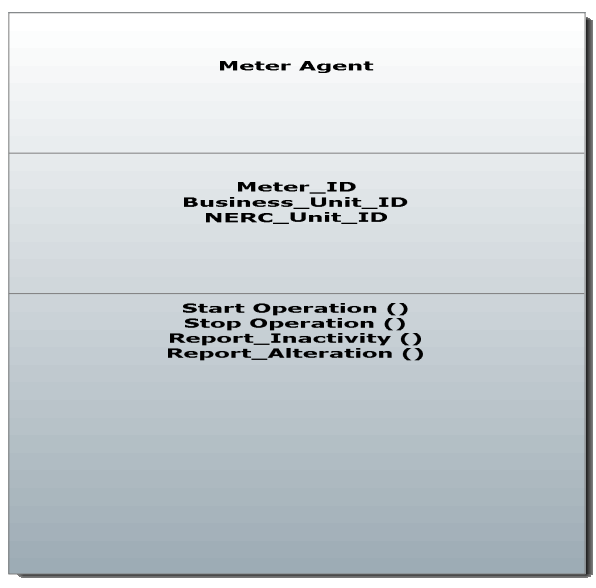

Figure 5. Meter Agent (Micro Level) Architecture.

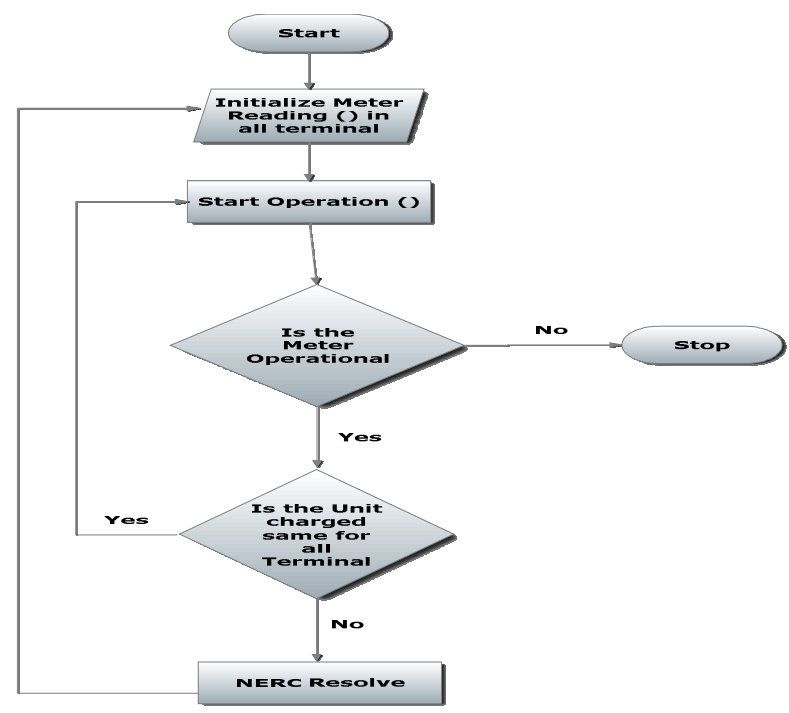

Figure 6. Flowchart of the Smart Metering Subsystem.

\subsection{Collaboration among Agents in MASs}

Our framework consists of the Switch Agent (SA), the Zone Agent (ZA), the Control Agent $\left(\mathrm{CA}_{\text {Master }}\right)$, Monitoring/FaultAgent (FA), Connection Agent (CA) and Meter Agent (MA). After 
the SA, ZA and the CA specifications, the next step involves the formalization of agent roles and the interactions applied to the problem at hand. To accomplish this task, a collaborative diagram which defines the interaction among agents and their interaction with the environment needed to be defined. The collaborative diagram of MAS is shown in Figure 7. The diagram illustrates how the FA, MA, SA, and CA interact together in the distributed system environment through their respective ZA. The interactions with the other committee of agents with the larger environment is through the overall Master Agent known as the CA Master. All message exchanges among agents are established via the Transmission Control Protocol/Internet Protocol or TCP/IP.

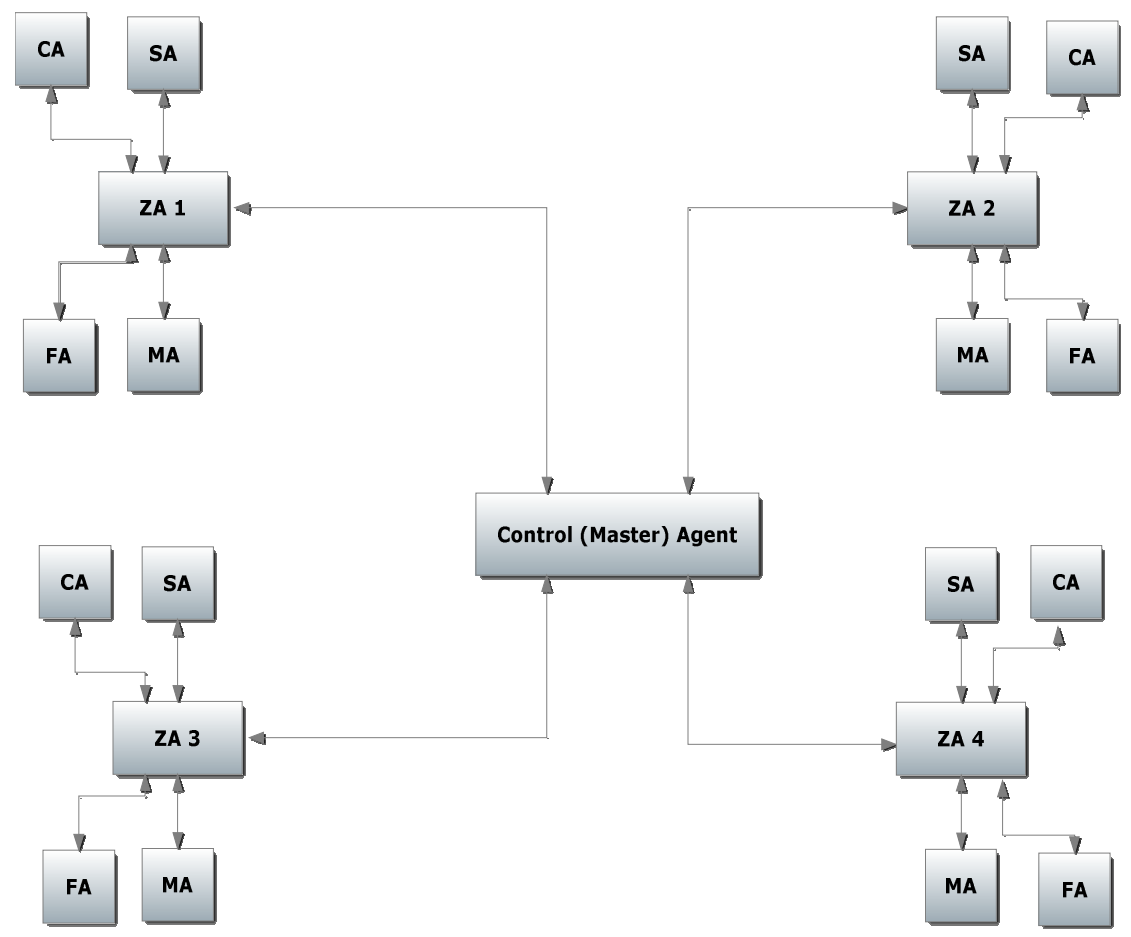

Figure 7: MAS Collaboration Diagram (Macro Level Architecture) of the extended Framework.

The initialization of the MAS is performed by the CA, the SA and the ZA notifying the CA of their presence. This includes notifying the CA of their names and IP addresses; the CA then updates its resource database and fulfills the required procedure for agent registration. Immediately, one of the functions in the Meter Agent known as the Start Operation is invoked. Whenever a fault occurs the FA sends a notification to the ZA. In order to demonstrate the proposed MAS, a prototype model is developed using Java Agent Development Framework.

\section{Results AND Discussion}

In order to demonstrate the proposed MAS, a prototype model was developed using Java Agent Development Framework. The prototype system work suitable in the proposed Architectural Framework is shown in Figure 8 below. The Architectural Framework demonstrates a fully distributed environment that comprises of NECR as the sole regulator of the Nigerian power sector and custodian of the proposed system, Distribution Company and Power consumer also known as customer. NERC and the Distribution Company interact together through their respective Server machines. While the power consumer meters communicate and interfaces with 
the NERC and Distribution Company through their State Offices and Business Unit respectively. That is to say any report send to the Distribution Company by the Customer Meter is being copied to the NERC at the same time.

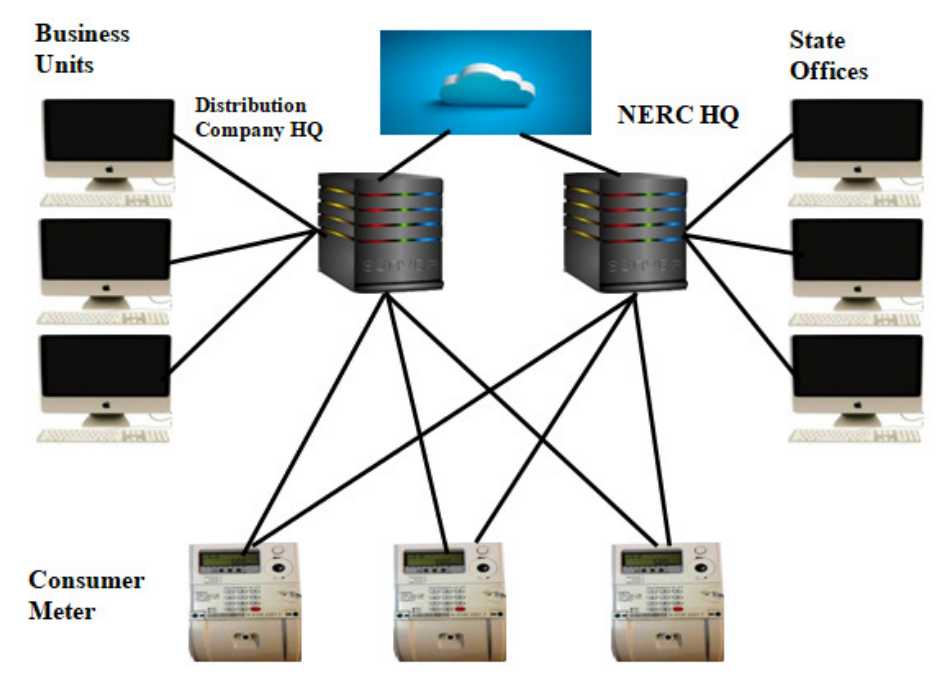

Figure 8. Architectural Framework Design of the Distributed Environment.

\section{Conclusions}

This paper presented the design and development of a Multi-Agent based framework for managing both Power Distribution activities and the regulation of the power sector. The main reason for proposing one distributed system for both NERC and Distribution Company is to ensure transparency in the sector. The distributed system will give the power regulators a clear picture of what is happening in the power sector. It will also help the Distribution Companies to address the power issues in the sector. It will at the same time give the customers a platform to channel their grievances. Due to the distributive nature of the power sector, which comprises of Regulators, Service providers and Consumers and Multi-Agent System been a distributed system itself, the system is capable of solving these problems affecting the Nigerian power sector. Furthermore as a future work, we shall look into the possibility of reducing the proximity for the various communication channels between the agents. We hope to achieve this by running more experiments using various feature selections to run complex classification algorithms that help identify the best features that predict optimized outcomes. This will also in the long run ensure the speedy response and acknowledgements within the system.

\section{REFERENCES}

[1] Adejumobi, I. A. and Pekum, A. J. (2009). "Software Application for Electrical Distribution System Reliability Studies: Box-Jenkins Methodology”. Pacific Journal of Science and Technology. 10(2):377-387.

[2] Bo, C. R. and Harry, H. (2010). "A Review of the Applications of Agent Technology in Trafficand Transportation Systems". IEEE Transactions on Intelligent Transportation Systems, Vol. 11, NO. 2, pp $44-49$. 
[3] Chinwuko, E. C., Nwuba, U. and Mgbemena, C. O. (2011). "Optimum Reliability and Cost of Power Distribution System: a case of Power Holding Company of Nigeria”. International Journal of Engineering Science \& Technology, 12(5): 233 - 238.

[4] Deshmukh, R. K., Davidson, E. M. and McArthur, S. D. J. (2008). "Exploiting Multi-Agent System technology within an autonomous regional active network management system”. Proceedings of the 14th IEEE International Conference on Intelligent Systems Application to Power Systems. Pp 57 - 61. New York.

[5] Dimeas, A. and Hatziargyriou, N. D. (2005). "Operation of a Multi-agent System for Micro Grid Control”. IEEE transaction on power systems, Vol. 20, No.3, 48 - 53.

[6] Ioannis, S. B. and Dimitris, P. L. (2007). "Implementing Multi-Agent Systems Technology for Power Distribution Network Control and Protection Management". IEEE Transactions on Power Delivery, Vol. 22, NO. 1, pp $14-19$.

[7] Maalal, S. and Addou, M. (2011). "A New Approach of Designing Multi-Agent Systems". International Journal of Advanced Computer Science and Applications, Vol.2, No. 11, pp 67 - 72.

[8] Rosa, M. A., Miranda, V., Carvalho, L. and DaSiva, A. M. L. (2010). Modern Computing Environment for Power System Reliability Assessment". Proceedings of the 12th ACM Probabilistic Methods Applied to Power Sytems, Pp 29 - 35. Singapore.

[9] Rosa, M. A., DaSilva, A. M. L., Miranda, V., Matos, M. and Sheblé, G. (2009). "Intelligent AgentBased Environment to Coordinate Maintenance Schedule Discussions". International Symposium on Intelligent Systems Applications to Power Systems, pp 22 - 27.

[10] Rumley, S., Elvira, K., Hugh, R. and Alain, G. (2010). "Multi-Agent Approach to Electrical Distribution Networks Control". 27th Annual IEEE International Computer Software and Applications Conference. Pp 62 - 68. New York

[11] Silaghi, G. C. (2005). "Software Engineering Approaches for Design of Multi- $\quad$ agent Systems". IEEE Transactions on Power Systems17(2): 457-462.

[12] Tao, Y., Nagata, T., Kimura, K., Sasaki, H. and Fujita, H. (2004). A Multi-Agent $\quad$ Approach to Distribution System Restoration". Proceedings of the 47th IEEE Midwest Symposium on Circuits and Systems, Vol. 2, pp $51-58$.

[13] Wang, F. Y. (2005). “Agent-Based Control for Networked Traffic Management Systems”. IEEE Intell. Syst., vol. 20, no. 5, pp. 92-96.

[14] Wan-Yu Yu, V., Men-Shen, T. and Yen-Bo, P. (2012). "Coordinating a Society of Switch Agents for Power Distribution Service Restoration in a Smart Grid". IEEE Trans. on Power System, Vol. 25, No. 1, pp. $72-81$.

[15] Zambonelli, F. and Parunak, H. V. D. (2003). "Signs of a Revolution in ComputerScience and Software Engineering”. In Proc. 3rd Int. Workshop Eng. Soc. Agents World, vol. 25, No. 9, pp. $13-21$.

[16] Ahmed M. K, Aliyuda A., and Bute M. S. (2017). Multi-Agent Based Monitoring and Control of Power Distribution System. In Proceedings of the 13th International Conference of Nigeria Computer Society. 
International Journal of Artificial Intelligence and Applications (IJAIA), Vol.10, No.1, January 2019

[17] Ali Aliyuda and Muhammed Kabir Ahmed. (2016): "Collaboration among Agents to Detect Fault in Power Distribution System" International Journal of Scientific \& Research Publications, Volume 6, Issue 8. 